\title{
Evaluation of Skeletal Hot Spots in Carcinoma Prostate Patients by Scintimetric Method \\ ${ }^{1}$ Afroza Naznin, ${ }^{2}$ Sadia Sultana, ${ }^{1}$ Kamrun Nahar, ${ }^{2}$ Taslima Sifat and ${ }^{2}$ Pupree Mutsuddy \\ ${ }^{1}$ Institute of Nuclear Medicine \& Allied Sciences (INMAS), Sylhet \\ ${ }^{2}$ National Institute of Nuclear Medicine \& Allied Sciences (NINMAS), Dhaka
}

Correspondence Address : Dr. Afroza Naznin, Medical Officer, Institute of Nuclear Medicine \& Allied Sciences (INMAS), Sylhet. M.A.G. Osmani Medical College Campus, P.O Box No-67, Sylhet-3100. E-mail: afroza.naznin@yahoo.com

\begin{abstract}
Skeletal scintigraphy is a highly sensitive tool in the detection of metastatic disease from prostate cancer, but its specificity is relatively low. Various quantitative parameters have been introduced to improve the bone scan specificity. Scintimetric method based Dr. V. Siva's retention ratio is one such parameter proposed to help in predicting malignant or metastatic nature of the skeletal hot spots in a bone scan non-invasively. This study was performed to evaluate the V. Siva's ratio in a small sample of prostate cancer patients in Bangladeshi population. This prospective observational study was carried out at the National Institute of Nuclear Medicine and Allied Sciences (NINMAS) from July 2015 to June 2016. A total of 32 patients with diagnosed prostate cancer were enrolled. Each patient underwent two bone scans at 4 hours and 24 hours respectively after radiopharmaceutical injection. Focal hot spots reported by expert Nuclear Medicine specialists were identified in both scans and maximum counts were taken by drawing region of interest over the spots. Dr. V. Siva's retention ratio was calculated by dividing the 4-hour count with 24 -hour count. Finally statistical analysis was done. The mean $\mathrm{V}$. Siva's ratio of metastatic group was $9.3 \pm 2.2(95 \% \mathrm{CI}$ 8.5-10.1) and that of degenerative group was $8.2 \pm$ 2.0 (95\% CI 7.5-8.9). Statistically significant difference was observed between the two groups. So it can be said that V. Siva's retention ratio might be useful as a quantitative parameter in adjunct to conventional bone scintigraphy for the skeletal survey of carcinoma prostate patients.
\end{abstract}

Key words: Prostate cancer, bone scan, hot spots, V. Siva's ratio.

\section{INTRODUCTION}

Carcinoma prostate is the second most common cancer in men worldwide. About 50\% patients have metastatic disease at the time of diagnosis and nearly all patients (85-100\%) who die of prostate carcinoma have skeletal metastases. The most commonly involved bones are vertebral column, ribs, skull and proximal ends of the long bones (1).
Skeletal scintigraphy shows very high sensitivity in the detection of metastatic disease from prostate cancer due to osteoblastic nature of the lesion (2). Bone scan requires a gamma camera and a bone seeking radiopharmaceutical e.g. ${ }^{99 \mathrm{~m}} \mathrm{Tc}$ hydroxymethylene diphosphonate (99mTc HMDP or HDP) and 99mTc methylene diphosphonate $\left({ }^{99 \mathrm{~m}} \mathrm{Tc} \quad \mathrm{MDP}\right)$. The radiopharmaceutical is incorporated into the bone mineral matrix of the region of metastatic involvement due to accelerated bone mineral turnover. As a result, an area of increased radiotracer uptake (hot spot) is seen. Even a $10 \%$ increase in bone mineral turnover in only a few millimeters sized area can be detected by bone scan, whereas plain radiographs require about $50 \%$ demineralization in a relatively large volume of bone. But despite its high sensitivity, bone scan often produces nonspecific or equivocal findings, because other types of lesion/damage to bone, e.g. degeneration or trauma can also result in a positive bone scan by turning on the osteoblasts and causing increased bone mineral turnover (3).

On the other hand, diagnosis of bone metastases is usually made by the presence of multiple focal lesions distributed randomly in the skeleton. But a solitary lesion in a patient with known malignancy has a significant chance of being metastatic. If the lesion is in the spine or pelvis, the risk of malignancy is about $60-70 \%$, in case of skull the risk is $40-50 \%$, and if in rib the chance of malignancy is 10 to $20 \%$. Other than being metastatic, a solitary abnormality also has the possibility to be a primary bone tumor, arthritis, trauma, 
enchondroma, osteoma, fibrous dysplasia, osteomyelitis or monostotic Paget disease (2). Moreover, the mean age of presentation for carcinoma prostate is 70 years which is also the usual age group for having degenerative change and fractures (4). To make an accurate interpretation in such diverse presentation, we take help from patient history and other investigations. Often the only certain way to establish the etiology is biopsy which is not only invasive but also may be very difficult to perform from a lesion in the axial skeleton. So it would be helpful if bone scintigraphy itself could provide a quantitative clue to the underlying pathology.

For a radionuclide bone scan ${ }^{99 \mathrm{~m}} \mathrm{Tc}$ MDP is administered intravenously, and then it undergoes protein binding in blood which increases to about $50 \%$ at 4 hours after injection. Only unbound tracers are available for bone uptake. 99mTc MDP undergoes passive diffusion through the capillary wall into the extravascular space and then binds to bone by physicochemical adsorption (chemisorption) to the hydroxyapatite structure of bone tissue. The net exchange of a substance between blood and tissue depends on blood flow, surface area and the capillary permeability (5). There are significant differences in the histological structure and metabolic handling of radiopharmaceuticals between the normal and diseased bone (6). This is what researchers tried to exploit in developing more objective methods to separate metastatic lesions from degenerative and other changes using bone scintigraphy.

Metastatic bone is mainly composed of woven bone which has a greater surface area than normal lamellar bone. Besides, the crystalline structure of hydroxyapatite in this newly formed bone is amorphous and biphosphonate compounds have significantly higher adsorption on amorphous calcium phosphate (6). This microarchitecture, high blood flow, and increased transcapillary migration all work together to result in rapid as well as intense radiotracer concentration in the metastatic lesions (7). But degenerative bone changes have increased amounts of lamellar bone which accounts for the relatively lower radiotracer uptake. This is supported by the work of Israel et al. (6), who found the 4-hour lesion-to-nonlesion uptake ratio in degenerative joint disease to be significantly lower than that of metastases. They showed that 24/4 hour ratio of ${ }^{99 m}$ Tc MDP uptake in bone metastasis and degenerative changes had significant different values and suggested that this ratio might be useful in cancer patients whose bone scans demonstrate solitary lesion. Later, scintimetric characterization by $4 / 24$ hour retention ratio was devised by Dr. V. Siva et al. (8). Bone scans were done in both anterior and posterior views at 4 and 24 hours. The focal hot spots in the processed images were identified and the maximum counts were calculated by drawing the region of interest over the skeletal hot spots. Then Dr. V. Siva's retention ratio was calculated by dividing the 4-hour count with 24hour count. It was found that malignant (metastatic) lesion showed the value greater than 10, degenerative lesions 5-10, and the benign lesions less than 5. This method was applied on delayed union of skeletal fractures where pathological fracture group obtained the mean value of $12.5 \pm 3.1$ and the benign group had the mean value of $6.68 \pm 2.8(9)$. Recently a study done on prostate cancer patients in Southern India found the mean of 4/24 hour Dr. V. Siva's retention ratio to be $11.5 \pm 2.8$ in case of metastatic disease from carcinoma prostate (8). This ratio might be a valuable noninvasive tool for identifying the underlying pathology, especially in doubtful cases.

This study was performed to evaluate the V. Siva's ratio in a small sample of prostate cancer patients in Bangladeshi population.

\section{PATIENTS AND METHODS}

A prospective observational study was carried out at the National Institute of Nuclear Medicine \& Allied Sciences (NINMAS) from July 2015 to June 2016. The population of this study was carcinoma prostate patients referred to NINMAS for bone scan. Patients with no hot spot in 4hour scan and patients having superscan appearance were excluded. A total of 32 patients with diagnosed prostate cancer were enrolled in this study. 
All the patients were informed of the potential risks and benefit of the procedure, and informed consent was taken from each individual case before inclusion in the study. Bone scintigraphy was performed with ${ }^{99 \mathrm{~m} T c}$ MDP given intravenously at a dose of 550-740 MBq $(15-20 \mathrm{mCi})$. Each patient underwent two bone scans at 4 hours and 24 hours respectively after radiopharmaceutical injection. Imaging was done by a dual head gamma camera using low energy, high resolution collimator. The whole body bone scanning was accomplished by obtaining anterior and posterior views. Focal hot spots reported by expert Nuclear Medicine specialists were identified in both scans and maximum counts were taken by drawing region of interest over the spots. Dr. V. Siva's retention ratio was calculated by dividing the 4-hour count with 24-hour count. Finally statistical analysis was done.

\section{RESULTS}

Total 32 carcinoma prostate patients age mean \pm SD, 67

\pm 8.1 (range $50-85$ years) were studied. Majority (43.8\%) of the patients belonged to age group 61-70 years. Among them, $14(43.8 \%)$ had skeletal metastasis, 6 (18.8\%) degenerative changes and $12(37.5 \%)$ had both metastatic and degenerative lesions according to conventional reports done by expert Nuclear Medicine physicians. Four patients $(12.5 \%)$ had solitary lesions and rest $(87.5 \%)$ had multiple site involvement.

Total 132 skeletal hot spots were identified, out of which 89 were reported as metastatic and 43 as degenerative. The mean V. Siva's ratio of metastatic group was $9.3 \pm 2.2(95 \%$ CI 8.5-10.1) and that of degenerative group was $8.2 \pm 2.0$ (95\% CI 7.5-8.9). Statistically significant difference was observed between the two groups (Table 1.)

Table 1: Comparison between Dr. Siva's retention ratio between metastatic and degenerative lesions

\begin{tabular}{llllll}
\hline Type of lesion & & \multicolumn{2}{l}{ V.Siva's ratio } & \multicolumn{1}{c}{$\mathbf{p}_{\text {value }}$} \\
\cline { 3 - 6 } & n & Mean \pm SD & & $\mathbf{9 5 \%}$ CI & \\
\cline { 3 - 6 } & & & Upper & Lower & \\
\hline Metastatic & 89 & $9.3 \pm 2.2$ & 8.5 & 10.1 & \multirow{2}{*}{0.006} \\
Degenerative & 43 & $8.2 \pm 2.0$ & 7.5 & 8.9 & \\
\hline
\end{tabular}

Among the four solitary lesions, three were metastatic and had the ratio above 10 . On the other hand, the only degenerative lesion was noted in left knee joint with V. Siva's ratio of 6.95 (Table 2.).

Table 2: Distribution of V. Siva's ratio in solitary lesion patients

\begin{tabular}{lll}
\hline Site of lesion & Type of lesion & V.Siva's ratio \\
\hline SI joint (left) & Metastatic & 10.62 \\
Rib & Metastatic & 10.30 \\
Thoracic vertebrae & Metastatic & 10.09 \\
Knee joint (left) & Degenerative & 6.95 \\
\hline
\end{tabular}

Seventeen out of 32 patients were symptomatic (complained pain) and 80 skeletal hot spots were reported in them. Among those, 61 were reported as metastatic and their mean V. Siva's ratio was $9.64 \pm$ 2.2. A total of 19 hot spots were reported as degenerative and their mean V. Siva's ratio was 8.69 \pm 2.3 . The difference was not statistically significant between two groups (Table 3 ).

Table 3: Distribution of the skeletal hot spots in symptomatic patients

\begin{tabular}{llll}
\hline Type of lesion & Number of lesions & $\begin{array}{l}\text { V.Siva's ratio } \\
(\text { Mean } \pm \text { SD) }\end{array}$ & p value \\
\hline Metastatic & 61 & $9.64 \pm 2.2$ & 0.1079 \\
Degenerative & 19 & $8.69 \pm 2.3$ & \\
\hline
\end{tabular}

Again, bone scans of 15 asymptomatic patients revealed 52 skeletal hot spots. Among those, 28 were reported as metastatic and their mean V. Siva's ratio was $8.69 \pm 2.2$. Degenerative hot spots were 24 in number and their mean V. Siva's ratio was $7.93 \pm 1.6$. The difference was not statistically significant between two groups (Table 4.).

Table 4: Distribution of the skeletal hot spots in asymptomatic patients

\begin{tabular}{llll}
\hline Type of lesion & Number of lesions & $\begin{array}{l}\text { V. Siva's ratio } \\
(\text { Mean } \pm \text { SD) }\end{array}$ & P value \\
\hline Metastatic & 28 & $8.69 \pm 2.2$ & 0.1667 \\
Degenerative & 24 & $7.93 \pm 1.6$ & \\
\hline
\end{tabular}

Total number of metastatic hot spots was 89 , out of which 28 were found in patients with S. PSA level less than or equal to $20 \mathrm{ng} / \mathrm{ml}$. Their mean V. Siva's ratio was 
$9.81 \pm 2.7$. Remaining 61 lesions were found in patients with S. PSA level > $20 \mathrm{ng} / \mathrm{ml}$. Their mean V. Siva's ratio was $9.13 \pm 2$. The difference was not statistically significant $(\mathrm{p}>0.05)$ between two groups (Table 5).

Table 5: V. Siva's ratio of metastatic hot spots in different S. PSA level

\begin{tabular}{llll}
\hline S. PSA $(\mathrm{ng} / \mathrm{ml})$ & $\begin{array}{l}\text { Number } \\
\text { metastatic lesions }\end{array}$ & $\begin{array}{c}\text { ofV. Siva's ratio } \\
\text { (Mean } \pm \text { SD) }\end{array}$ & p value \\
\hline$\leq 20$ & 28 & $9.81 \pm 2.7$ & $0.1872^{\text {ns }}$ \\
$>20$ & 61 & $9.13 \pm 2$ & \\
\hline
\end{tabular}

No correlation was found between S. PSA level and V. Siva's ratio of the study patients (Figure 1).

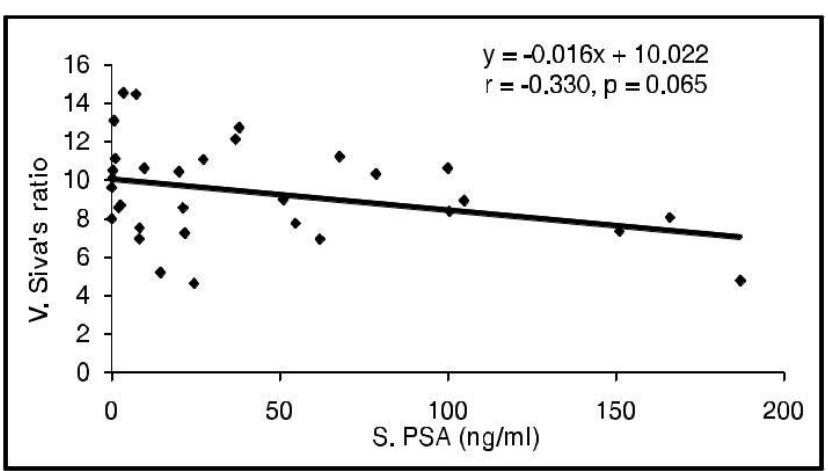

Figure 1: Scatter diagram showing no correlation between S. PSA (ng/ml) and V. Siva's ratio of the study patients.

\section{DISCUSSION}

In the present study mean V. Siva's retention ratio of metastatic group was 9.3 and that of degenerative group was 8.2. The difference between the two groups was statistically significant. Among the four solitary lesions, three were metastatic and their V. Siva's ratios were all above 10 . On the other hand, retention ratio of the solitary degenerative lesion was 6.95. In a previous study, Sivasubramaniyan and Venkataramaniyah (8) characterized 101 focal skeletal hot spots in 16 biopsy proven carcinoma prostate patients using the same procedure. It was also applied in 92 lesions in 32 known benign disease patients. The mean value of $4 / 24$ hour Dr. V. Siva's retention ratio was found to be 11.5 in the carcinoma prostate group and 4.8 in the benign group. Findings in the present study though statistically significant, are lower than their obtained values. The reason behind this mild discrepancy may be due to smaller sample size in this study. However, findings in case of solitary lesions are in concordance with the proposed range of malignant and benign lesions in scintimetric method based V. Siva's ratio (9).

V. Siva's ratio has been found to be slightly altered when other complications are present with skeletal metastases. It was explored by Namdeo et al. (9) for assessing fracture complications in a study involving 30 cases of delayed / non-union of skeletal fractures. The mean V. Siva's ratio in the metastatic pathological fracture group was 12.5. On the other hand, benign fracture group with complications like stress fracture and benign bone tumors showed the mean value of 6.68. Reason behind the higher values in their study may be the additional osteoblastic activity of fracture complication on top of metastatic change in bone. But this aspect was not included in the present study because none of the study subjects had any evidence of fracture.

Out of total 32 patients in this study, 17 were symptomatic. Their bone scans showed total 80 skeletal hot spots. Among those, 61 were reported as metastatic and their mean V. Siva's ratio was 9.64. Degenerative hot spots were 19 in number and their mean V. Siva's ratio was 8.69. The difference was not statistically significant ( $p>0.05$ ) between two groups. Again, bone scans of 15 asymptomatic patients revealed 52 skeletal hot spots. Among those, 28 were reported as metastatic and their mean V. Siva's ratio was 8.69. Degenerative hot spots were 24 in number and their mean V. Siva's ratio was 7.93. The difference was not statistically significant $(\mathrm{p}>0.05)$ between two groups. Fallahi et al. (10) studied the distribution pattern and imaging characteristics of bone metastases detected by conventional bone scan in patients with different types of malignancies to assess their relationship with the complaint of bone pain. No significant association was noted between the site of bone metastases and pain perception in prostate cancer patients. The relation between bone pain and scintimetric character of skeletal 
lesion has not been addressed yet. Bone pain is a nonspecific symptom and may be caused by both metastasis as well as degenerative disease. This might be the reason behind the lack of significant difference in V. Siva's ratios when observed in context of pain.

In the current study, total number of metastatic hot spots was 89 , out of which 28 were found in patients with $\mathrm{S}$. PSA level less than or equal to $20 \mathrm{ng} / \mathrm{ml}$. Their mean V. Siva's ratio was 9.81. Remaining 61 lesions were found in patients with S. PSA level of $>20 \mathrm{ng} / \mathrm{ml}$. Their mean V. Siva's ratio was 9.13. The difference was not statistically significant $(\mathrm{p}>0.05)$ between two groups. S. PSA is a widely accepted biochemical marker for carcinoma prostate. Its serum level depends on tumor size, differentiation rate and stage (11). Higher PSA level also correlates with bone metastasis (12). But PSA is normally produced by prostatic epithelial cells, and when neoplastic tissue invades the prostatic capsule large quantity of PSA is shed into the bloodstream. So higher S. PSA level may predict presence and extent of skeletal metastasis, but it is not directly related to osteoblastic activity in bone. Probably this is why no correlation was found between $\mathrm{S}$. PSA and V. Siva's ratio of the study patients in the current study.

\section{CONCLUSION}

Management protocol for patients with prostate cancer depends to a large extent on the absence or presence of skeletal metastases. Imaging of metastases in carcinoma prostate is often a challenge and even though there are currently several new imaging modalities for the detection of skeletal metastases, the age old nuclear medicine bone scintigraphy has proved to be the mainstay due to its high sensitivity, low cost and easy availability. However, it suffers from relative low specificity and the differentiation of true metastasis from a benign bone lesion is sometimes very difficult. Dr. V. Siva's retention ratio for this purpose was reported to be useful. In the present study, the same V. Siva's retention ratio protocol applied on a small number of prostate cancer patients in Bangladesh gave promising results.
The V. Siva's retention ratio of metastatic skeletal hot spots was found to be 9.3 and that of degenerative hot spots 8.2. The difference was statistically significant. Therefore, it may be concluded that the inclusion of $\mathrm{V}$ Siva's quantitative parameter in adjunct to conventional bone scintigraphy for the skeletal survey of carcinoma prostate patients can help to narrow down the specificity spectrum of bone scan hot spots being either due to metastasis versus benign disease.

\section{REFERENCES}

1. Carlin BI and Andriole GL. The natural history, skeletal complications, and management of bone metastases in patients with prostate carcinoma. Cancer Supplement 2000;88:2989-2994.

2. Ziessman HA, O’Malley JP and Thrall JH. Nuclear Medicine: The Requisites. 4th ed. Pennsylvania: Elsevier Saunders, 2014:98-130.

3. Imbriaco M, Larson SM, Yeung HW, Mawlawi OR, Erdi Y, Venkatraman ES and Scher HI. A new parameter for measuring metastatic bone involvement by prostate cancer: the Bone Scan Index. Clin Cancer Res 1998;4:1765-1772.

4. Goddard J, Turner AN, Cumming AD and Stewart LH. Kidney and urinary tract disease. In: Boon NA, Colledge NR, Walker BR and Hunter JAA, editors. Davidson's Principles \& Practice of Medicine. 23rd ed. India: Elsevier, 2006:462-523.

5. Wong KK and Piert M. Dynamic bone imaging with $99 \mathrm{~m} \mathrm{Tc}$ labeled Diphosphonates and 18F-NaF: mechanisms and applications. J Nucl Med 2013;54:590-599.

6. Israel O, Front D, Frenkel A and Kleinhaus U. 24 hour/4 hour ratio of Technetium-99m Methylene Diphosphonate uptake in patients with bone metastases and degenerative bone changes. J Nucl Med 1985;26:237-240.

7. Castronovo FP, Guiberteau MJ, Berg G, Mckusick KA, Callahan RJ and Potsaid MS. Pharmacokinetics of Technetium-99m Diphosphonate. J Nucl Med 1977;18:809-814.

8. Sivasubramaniyan $\mathrm{V}$ and Venkataramaniah K. Temporal scintimetric characterization of skeletal hotspots in bone scan by Dr. V. Siva's retention ratio. In: Satapathy SC, Mandal JK, Udgata SK and Bhateja V, editors. Information Systems Design and Intelligent Applications, Advances in Intelligent Systems and Computing. India: Springer, 2016:297-306.

9. Namdeo R, Sivasubramaniyan V, Sai NV and Venkataramaniah K. Scintimetric evaluation in the assessment of delayed union of skeletal fractures. Int J Innov Res \& Dev 2015;4:362-365.

10. Fallahi B, Beiki D, Khodaparast MB, Gholamrezanezhad A, FardEsfahani A and Eftekhari M. Additive clinical value of bone scintigraphy in patients with malignant tumors with absence of localized bone pain: A report on most common sex-related cancers. Iranian J Nucl Med 2011;19:51-58.

11. Ugrinska A, Bomberdieri E, Stokkel MPM, Crippa E and Pauwels EKJ. Circulating tumor markers and nuclear medicine imaging modalities: breast, prostate and ovarian cancer. Q J Nucl Med 2002;46:88-104.

12. Ozu C, Nakashima J, Horiguchi Y, Oya M, Ohigashi T and Murai M Prediction of bone metastases by combination of tartrate-resistant acid phosphatase, alkaline phosphatase and prostate specific antigen in patients with prostate cancer. Int J Urol 2008;15:419-422. 